
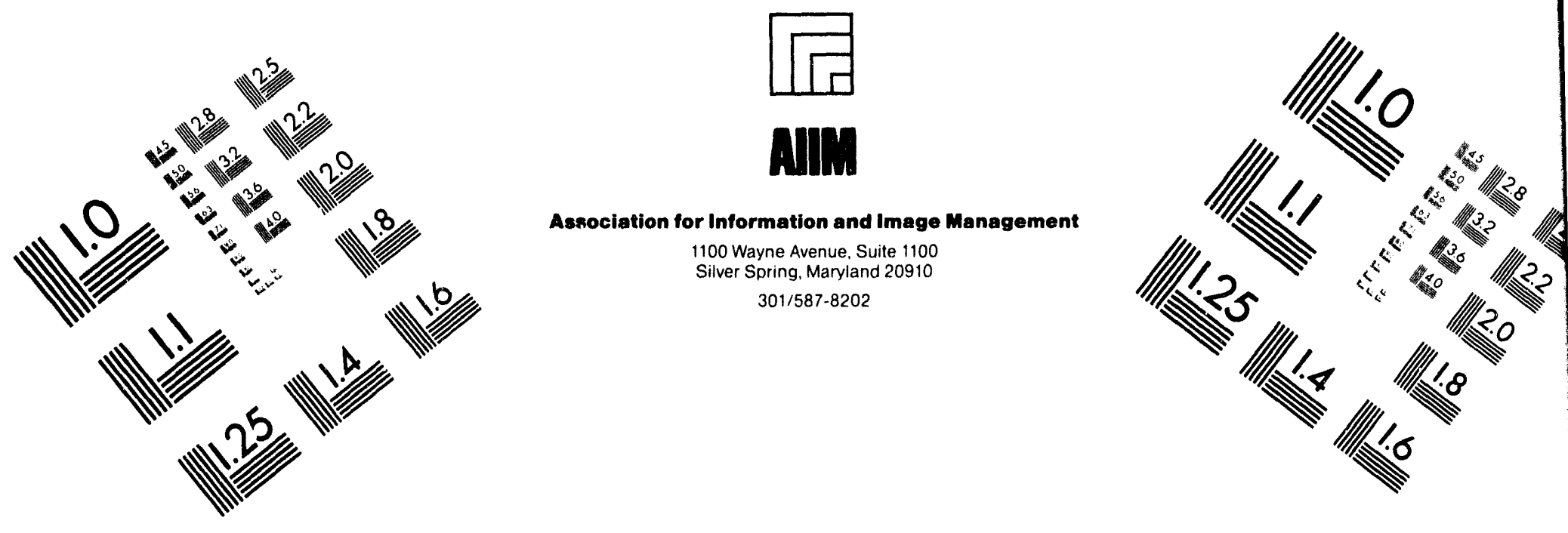

\title{
Centimeter
}

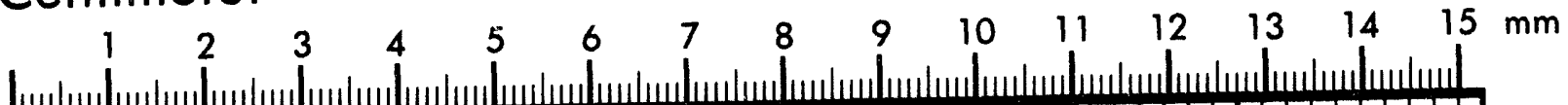
$\mid$ Inches
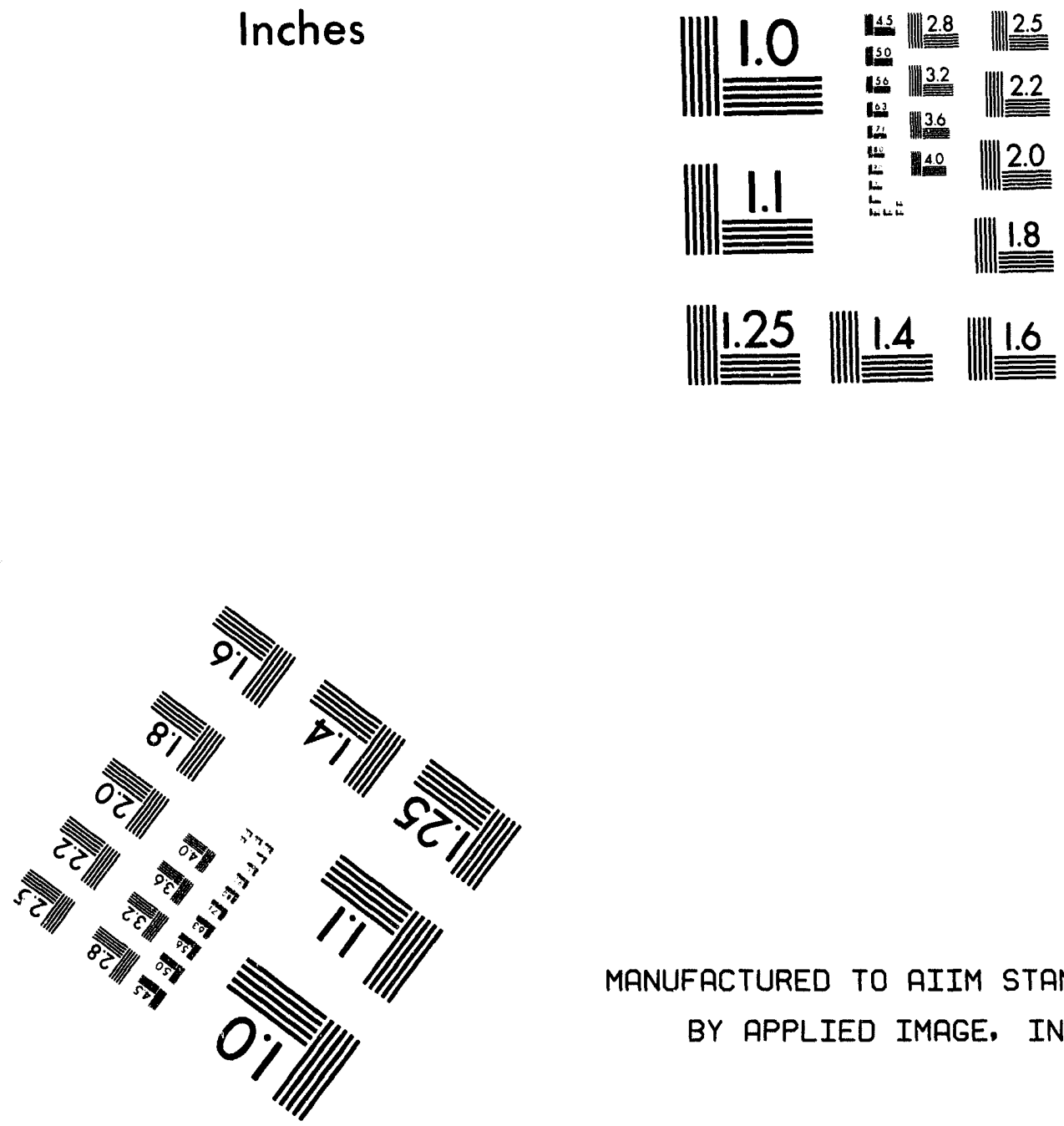

MANUFACTURED TO AIIM STANDARDS BY APPLIED IMAGE, INC.

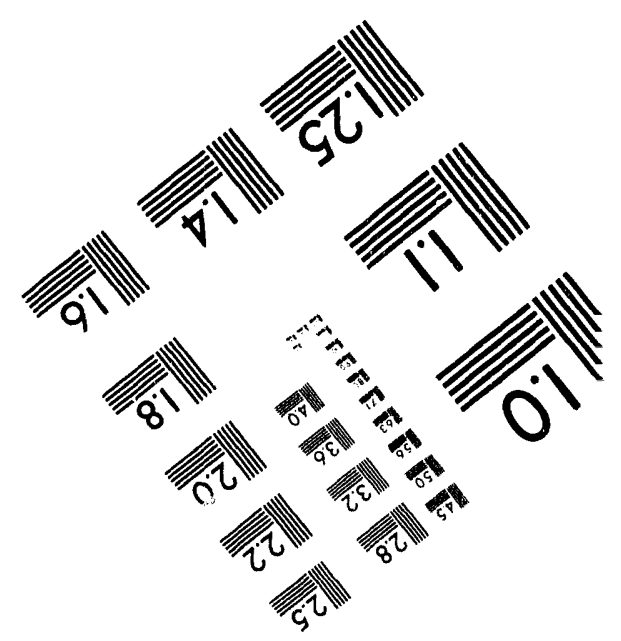



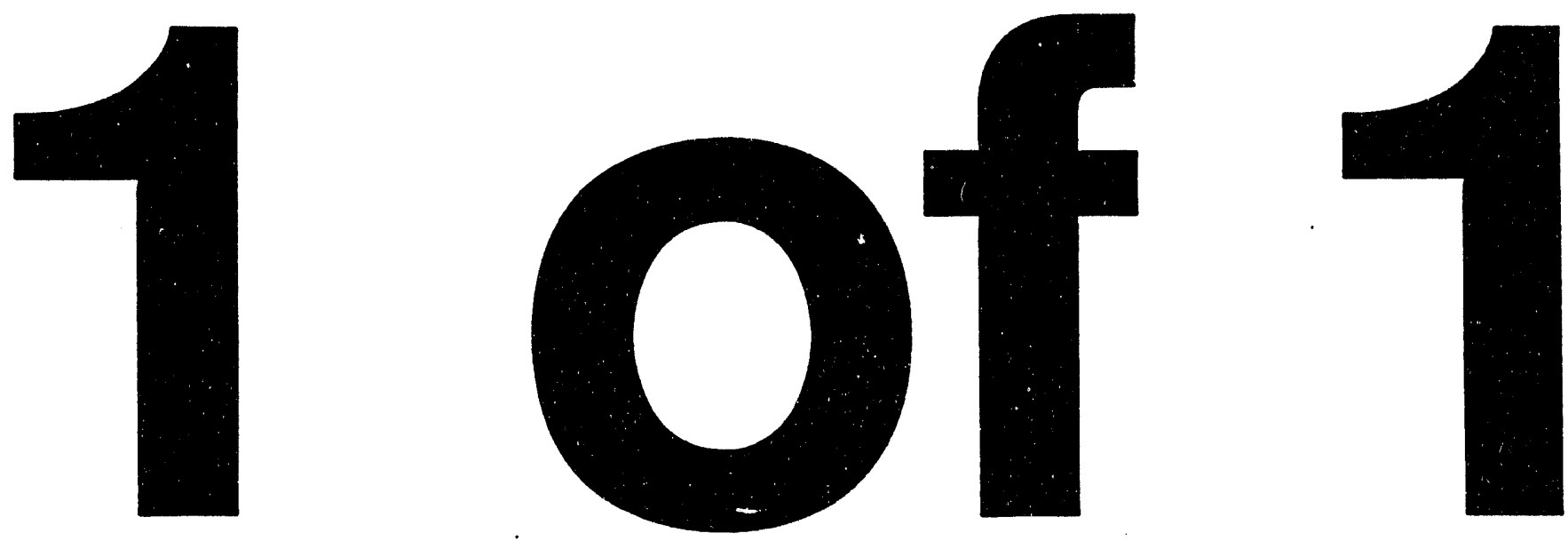
DOE/ER/13519-9

\section{EXPERIMENTAL STUDY OF INTERACTIONS OF HIGHLY CHARGED IONS WITH ATOMS AT keV ENERGIES}

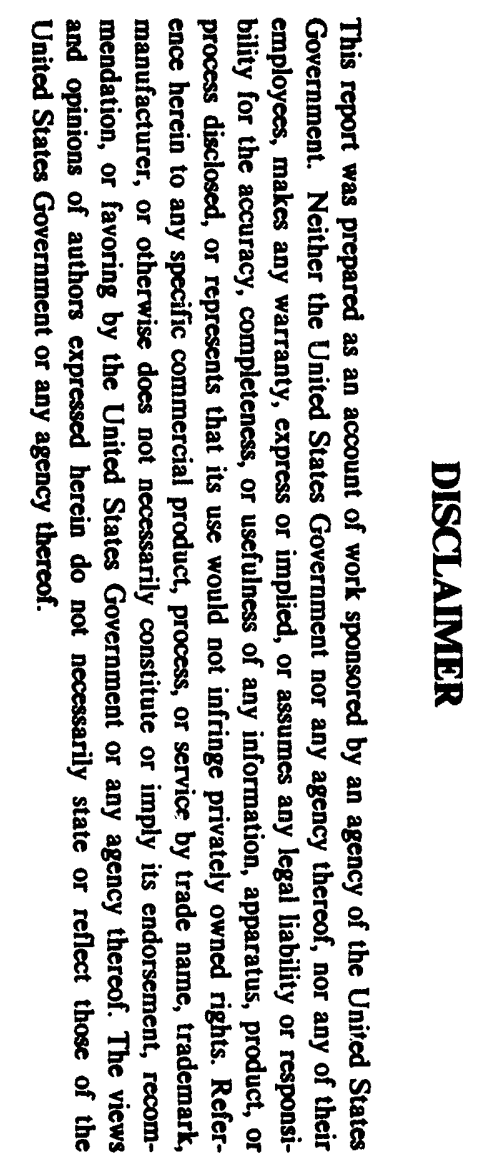

Progress Report

for the Period

April 16, 1994 - August 1, 1994

V.O. Kostroun

Cornell University

Ithaca, New York 14853

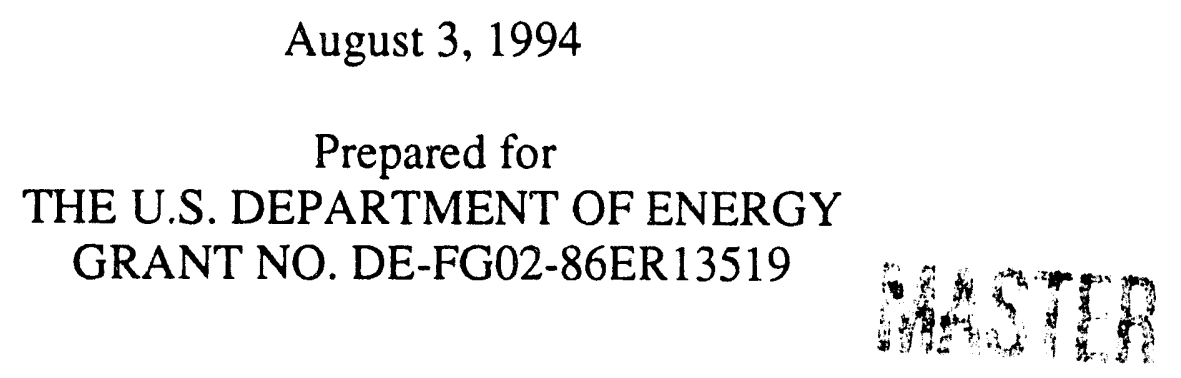

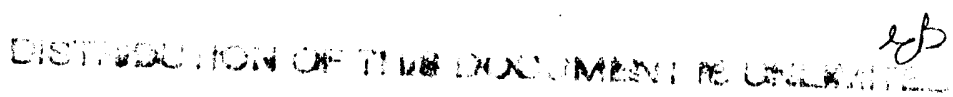




\section{Introduction}

The objective of this program is the experimental study of interactions of low energy, highly charged ions with other atomic species. The Cornell superconducting solenoid, cryogenic electron beam ion sourc CEBIS $^{1}$ designed and built in our laboratory is the major piece of apparatus used in these investigations. This progress report describes the work accomplished during the period April 16, 1994 and August 1, 1994. This includes both finished experiments and preparatory work for planned future experiments using the source.

\section{Experimental work finished}

During this time, we have completed measurements of the angular distributions and energy gains in $\mathrm{Ar}^{\mathrm{q}}(11 \leq \mathrm{q} \leq 14)$ on $\mathrm{Ar}$ collisions at $72 \mathrm{qeV}$ laboratory energy. In particular, energy gain spectra at different laboratory scattering angles were obtained for $\operatorname{Ar}(q-1)+$ projectiles, i.e. projectiles whose final charge state had decreased by one unit. The experimental technique used, and the method of analysis are described elsewhere. 2,3 The raw spectra are similar to those observed for $\mathrm{Ar}^{8+}$ and $\mathrm{Ar}^{10+}$ on $\mathrm{Ar}$ at comparable energies ${ }^{3}$, as well as those described in the last progress report for $\mathrm{Ar}^{12+}$ on $\mathrm{Ar} .{ }^{4}$ Figure 1 shows the scattering diagrams constructed from the data according to the discussion presented in reference 3 .

The data exhibit the odd-even effect described in an earlier paper. ${ }^{5}$ That is, odd charge argon projectiles incident on Ar show only one distinct peak in the zero degree energy gain spectra, and even charge projectiles two peaks. For both even and odd projectile charge states, the scattered one electron transfer projectile energy gain spectra show a broad "peak" at higher energy gains. The angular distributions of scattered projectiles whose energies are associated with the different "peaks" in the energy gain spectra are quite distinct. The angular distributions corresponding to the lower energy gain peaks are fairly narrow, and as the energy gain increases, so does the overall width of the angular distribution. The situation is similar to that for $\mathrm{Ar}^{12+}$ described in the last Progress Report. 4

The energy gain spectra obtained at different laboratory scattering angles $\theta$ were converted into Q-value spectra using the relation 2

$$
\mathrm{Q}=\frac{\left(\mathrm{m}_{\mathrm{l}}+\mathrm{m}_{\mathrm{p}}\right)}{\mathrm{m}_{\mathrm{t}}} \Delta \mathrm{T}+\frac{2 \mathrm{~m}_{\mathrm{p}}}{\mathrm{m}_{\mathrm{t}}}\left[1-\sqrt{1+\frac{\Delta \mathrm{T}}{\mathrm{T}_{\mathrm{p}}}} \cos \theta\right] \mathrm{T}_{\mathrm{p}}
$$

where $T_{p}$ is the projectile energy and $\Delta T \equiv T_{p}^{\prime}-T_{p}$ is the scattered projectile energy gain. In deriving this expression, one makes use of the definition of the $Q$ value in the laboratory system

$$
Q=\left(T_{p}^{\prime}+T_{t}^{\prime}\right)-\left(T_{p}+T_{l}\right)=\Delta T+\left(T_{t}^{\prime}-T_{l}\right)
$$

where $T_{t}$ is the initial, and $T_{t}$ the final kinetic energy of the target.

Once the energy gain data at different laboratory scattering angles were converted to Q-value data, the angular distributions were transformed to the $\mathrm{cm}$ system, figure 1 , and integrated over $\mathrm{cm}$ 
scattering angles to give $P(Q)$ vs $Q$ curves, figure 2 .
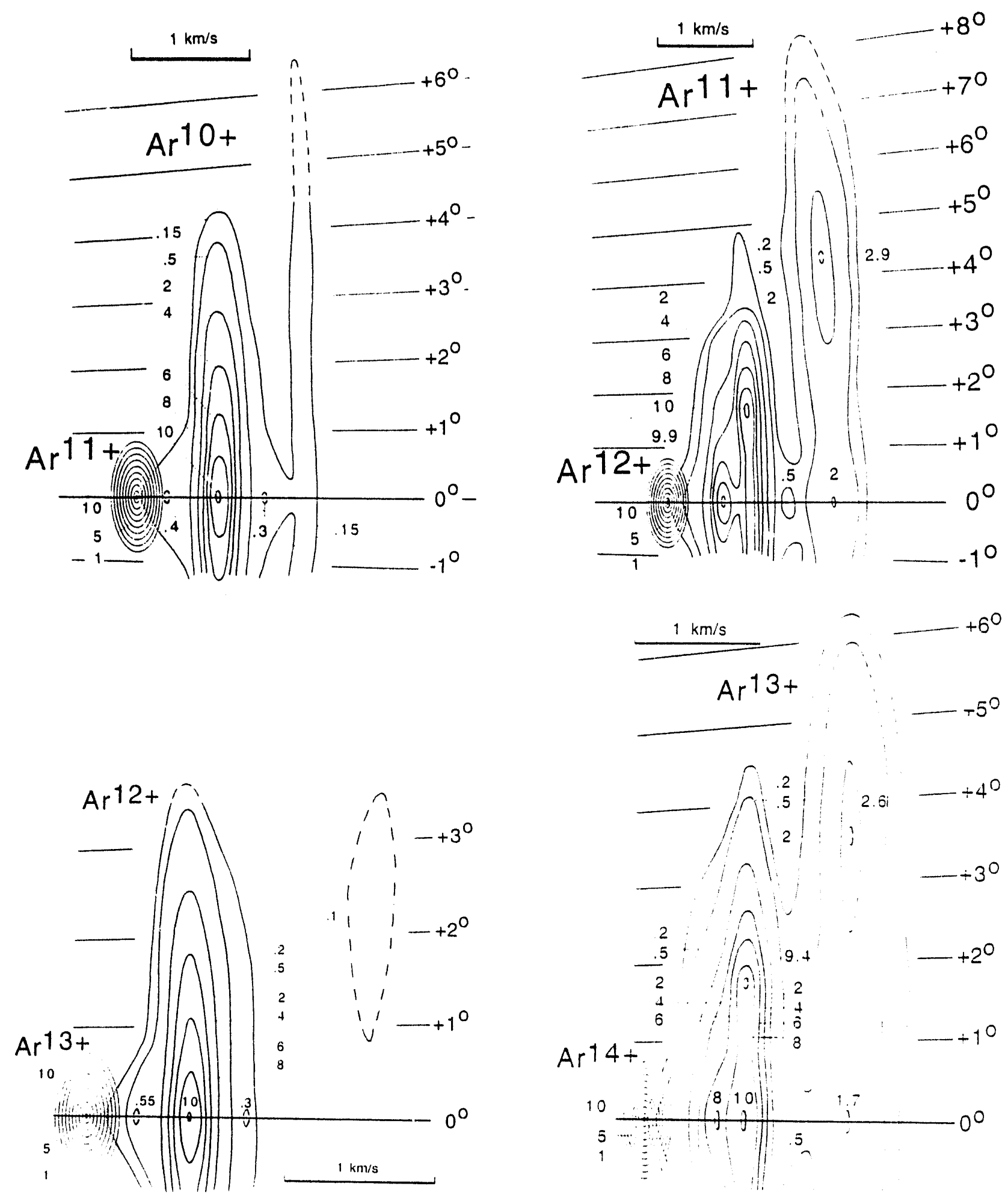

Figure 1. Scattering diagrams for $\mathrm{Ar}^{\mathrm{q}+}(11 \leq \mathrm{q} \leq 14)$ on Ar collisions at $72 \mathrm{qeV}$ laboratory energy. 

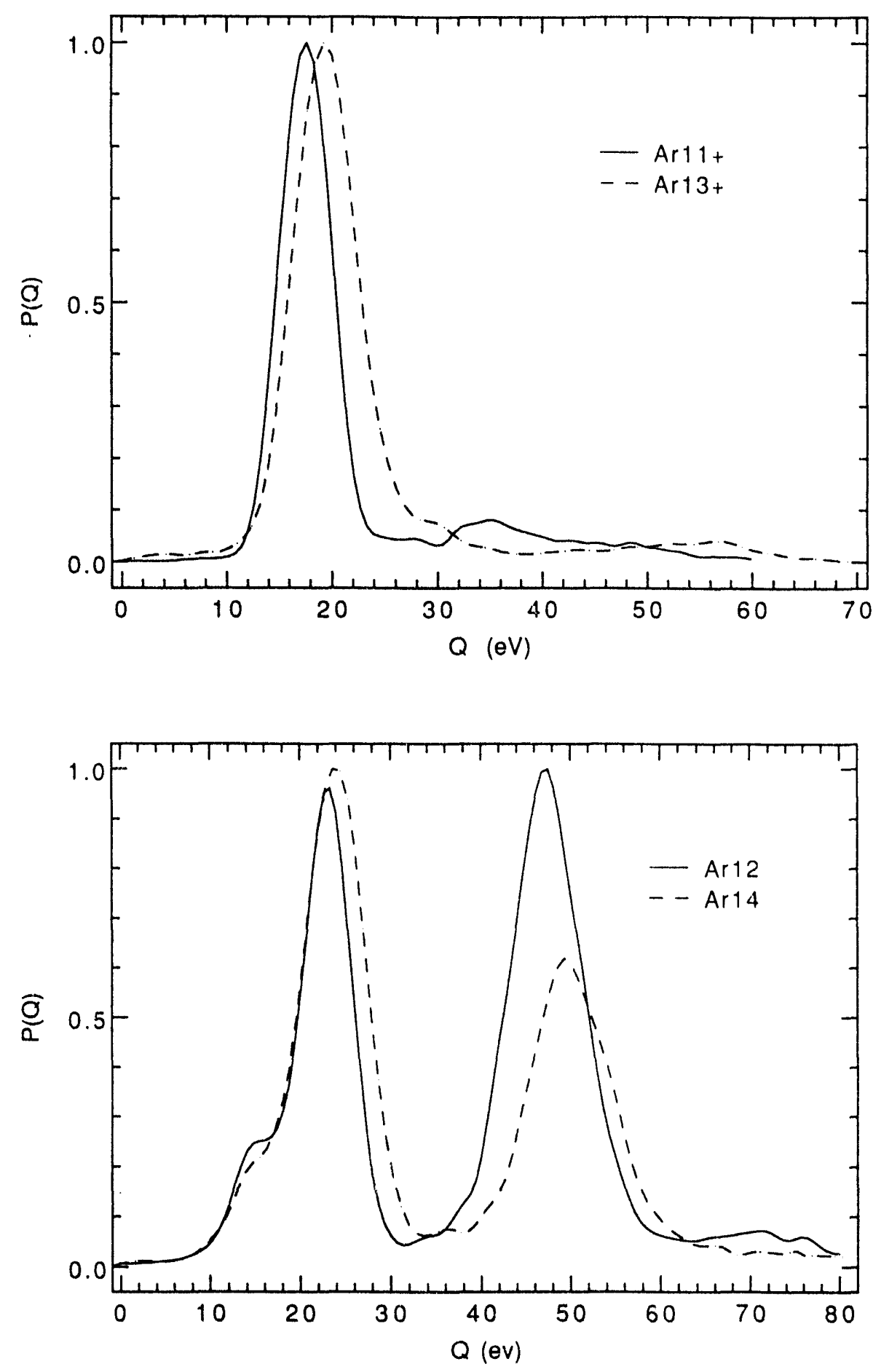

Figure 2. Scattering diagrams in figure 1 integrated over $\mathrm{cm}$ scattering angles. The resulting $P(Q)$ vs $Q$ curves reflect the density of capture states as a function of the inelastic energy change $Q$ in the collision. 
The above results, figure 2 , are quite puzzling, and not completely understcod at this point. An article based on the work is in preparation.

\section{Work in progress}

Some time ago we had construrted a microwave discharge cavity to produce atomic hydrogen. The cavity operates at $2.45 \mathrm{GHz}$, and is based on the Evenson design. ${ }^{6}$ The $\lambda / 4$ Evanson cavity was extended by three wavelengths $\lambda$ as described by Murphy and Brody. 7 The cavity microwave power supply was constructed using a magnetron from a commercially available microwave oven. Figure 3 shows some very preliminary results on the discharge spectrum measured with a $1 / 4 \mathrm{~m}$ Jarrell-Ash monochromator. The hydrogen Balmer series is clearly visible. (The spectral response of the 1P28 photomultiplier used to record the spectrum drops off very rapidly above $5200 \AA$, and hence the Balmer $\alpha$ is quite weak.) At the higher discharge pressure, molecular hydrogen is clearly present. To measure the dissociation fraction, we have placed a quadrupole mass analyzer $25 \mathrm{~cm}$ below the nozzle of the discharge. Without any HF treatment of the Vycor glass, the dissociation fraction was $40 \%$. Efforts are under way to find the best operating conditions of the source and to improve the dissociation fraction to $90 \%$ or better.

The microwave discharge cavity will be used to produce atomic hydrogen, oxygen and nitrogen targets for collision studies involving low energy, highly charged ions and these species.

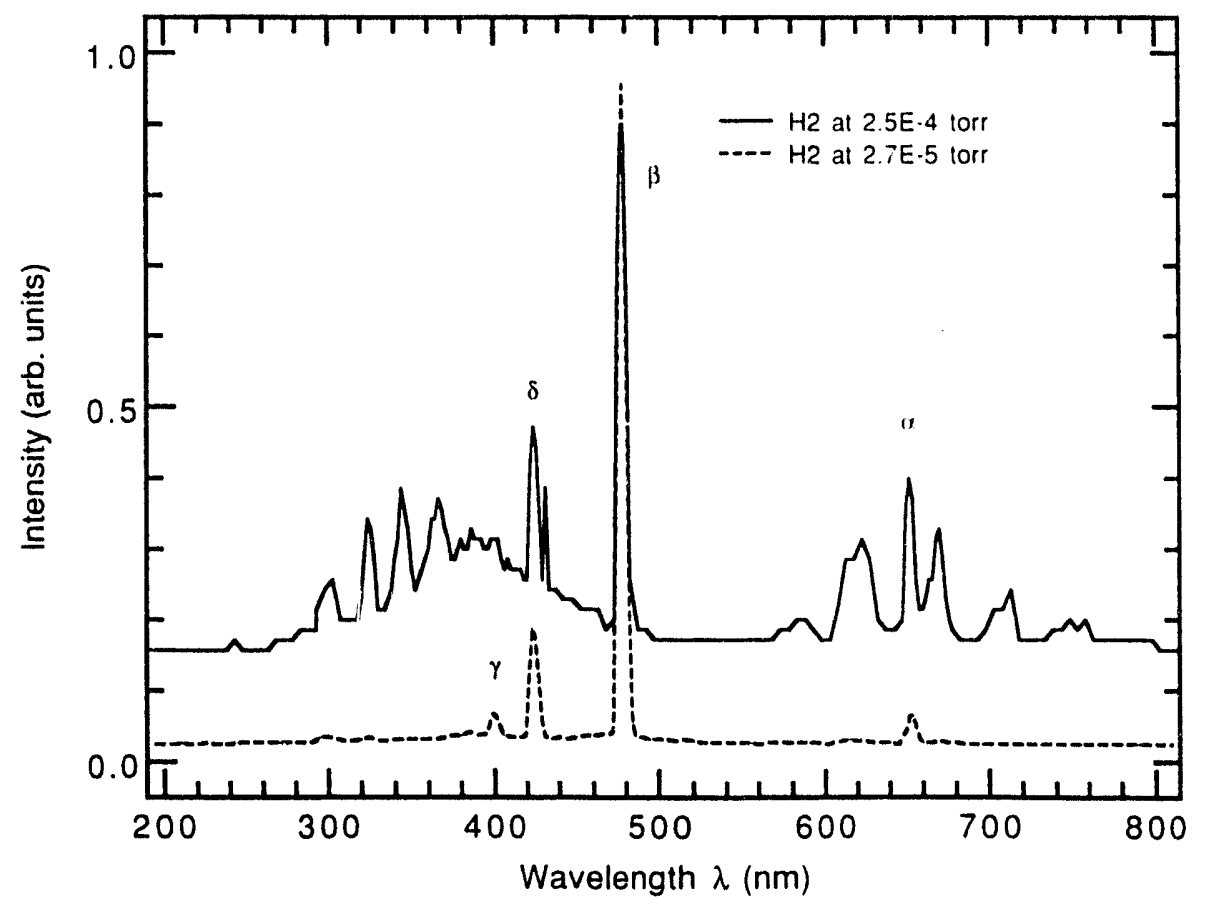

Figure 3. Hydrogen spectrum in the visible produced by the $2.45 \mathrm{GHz}$ microwave discharge source described above. At a vacuum system background pressure of $2.5 \times 10^{-4} \mathrm{Torr}$, the spectrum shows a distinct molecular component. At a background pressure ten times lower, only atomic hydrogen is visible. 
In addition to the above wurk, we have obtained some preliminary measurements on the angular distribution of ions scattered from a Si $<111>$ crystal, as well as the energy distribution of particles coming off the surface at a given projectile angle of incidence. These exploratory measurements give us some idea as to the feasibility of a certain class of experiments involving low energy, highly charged ions incident on surfaces. The experimental arrangement is shown in figure 4 .
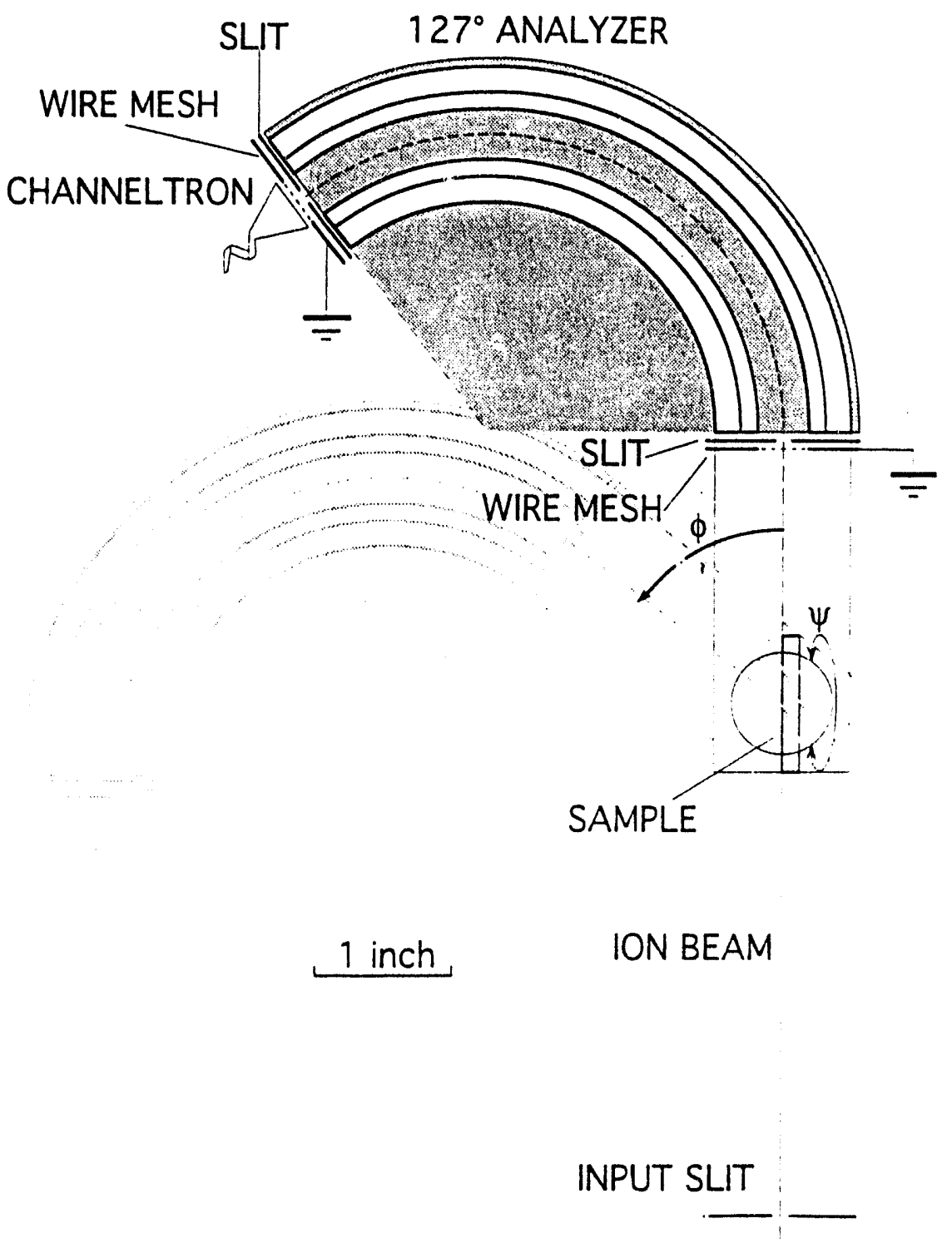

Figure 4. Experimental arrangement used to measure the energy and angular distribution of ions scattered from surfaces.

In this particular experiment, $2.3 \mathrm{qkeV} \mathrm{Ar}^{8+}$ ions impinged on a $\mathrm{Si}<111>$ surface at $7.5^{\circ}$. The pressure in the vacuum chamber was $1.0 \times 10^{-8}$ Torr. To remove the $\mathrm{SiO}_{2}$ layer, the crystal 
was bombarded in situ for ten minutes by a 10 microampere, $3 \mathrm{keV} \mathrm{Ar}{ }^{+}$beam produced by a PIG source. The total fluence was $3.6 \times 10^{16}$ ions $/ \mathrm{cm}^{2}$. Figure 5 shows the angular distribution of particles, regardless of species, energy or charge, emitted from the surface.

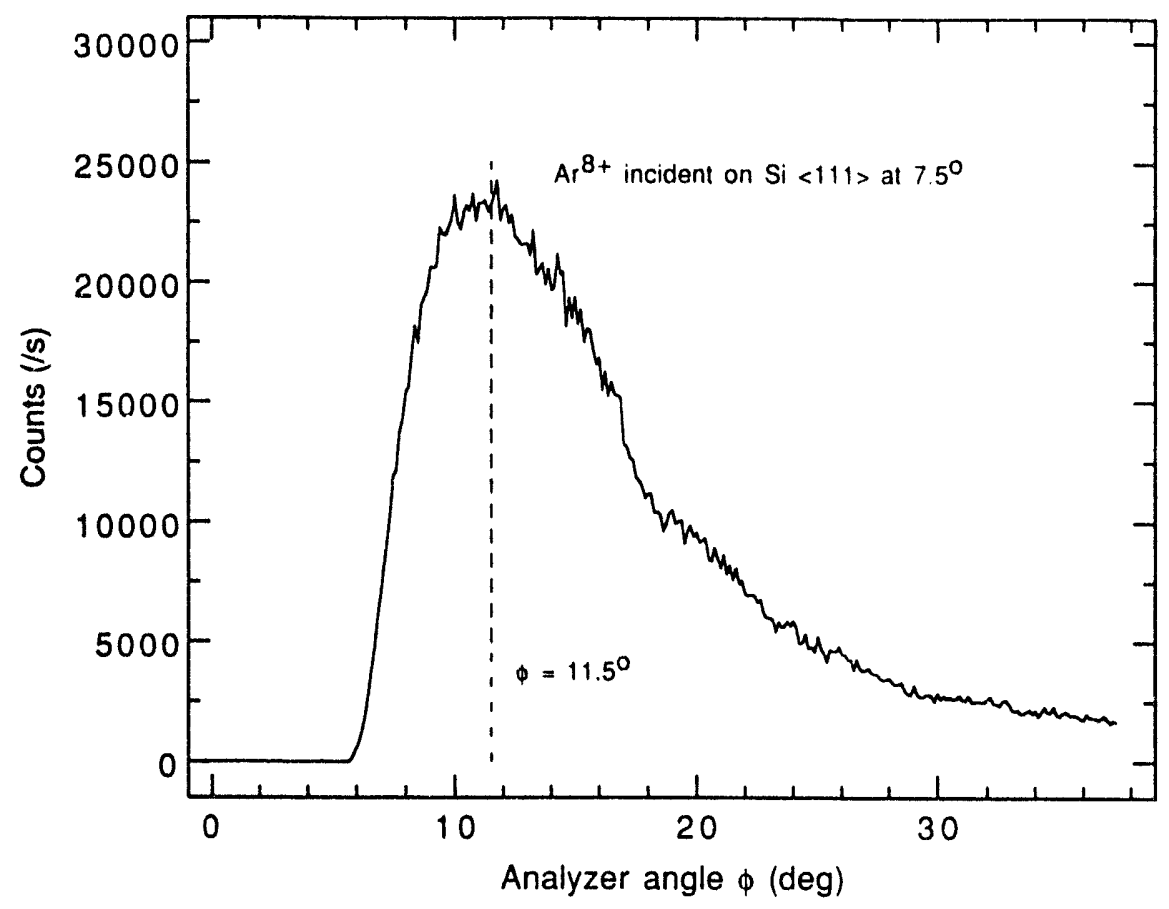

Figure 5. Angular distribution of particles, regardless of species, energy or charge, emitted when $2.3 \mathrm{qkeV} \mathrm{Ar}{ }^{8+}$ ions impinged on a $\mathrm{Si}<111>$ surface at $7.5^{\circ}$.

The spectrum was obtained as follows. The $127^{\circ}$ electrostatic analyzer has a hole (covered by a wire mesh) in its outer electrode (not shown in figure 4). This allows particles to pass through the hole along a line perpendicular to the entrance slit, when no potential is applied to the analyzer electrodes. A channeltron mounted behind the hole in the outer electrode detects particles that pass through the analyzer entrance slit. Both charged and neutral particles (if energetic enough) are detected. By rotating the analyzer and recording the detected particles as a function of the analyzer angle $\phi$, we obtained the above figure. In figure 6 , the $\mathrm{Ar}^{8+}$ impinged on the $\mathrm{Si}\langle 111\rangle$ surface at $7.5^{\circ}$, and the electrostatic analyzer was set at $11.5^{\circ}$, the maximum in the curve shown in figure 5 . The $127^{\circ}$ analyzer voltage was then scanned, and the positively charged particles that made it through the analyzer recorded.

The electrostatic analyzer was scanned as follows. The analyzer is electrically isolated from ground. By applying a fixed potential difference $(12 \mathrm{~V})$ to the analyzer electrodes, and varying the potential at which the analyzer floats, i.e. the potential along the central orbit, one can record spectra at constant transmission. 


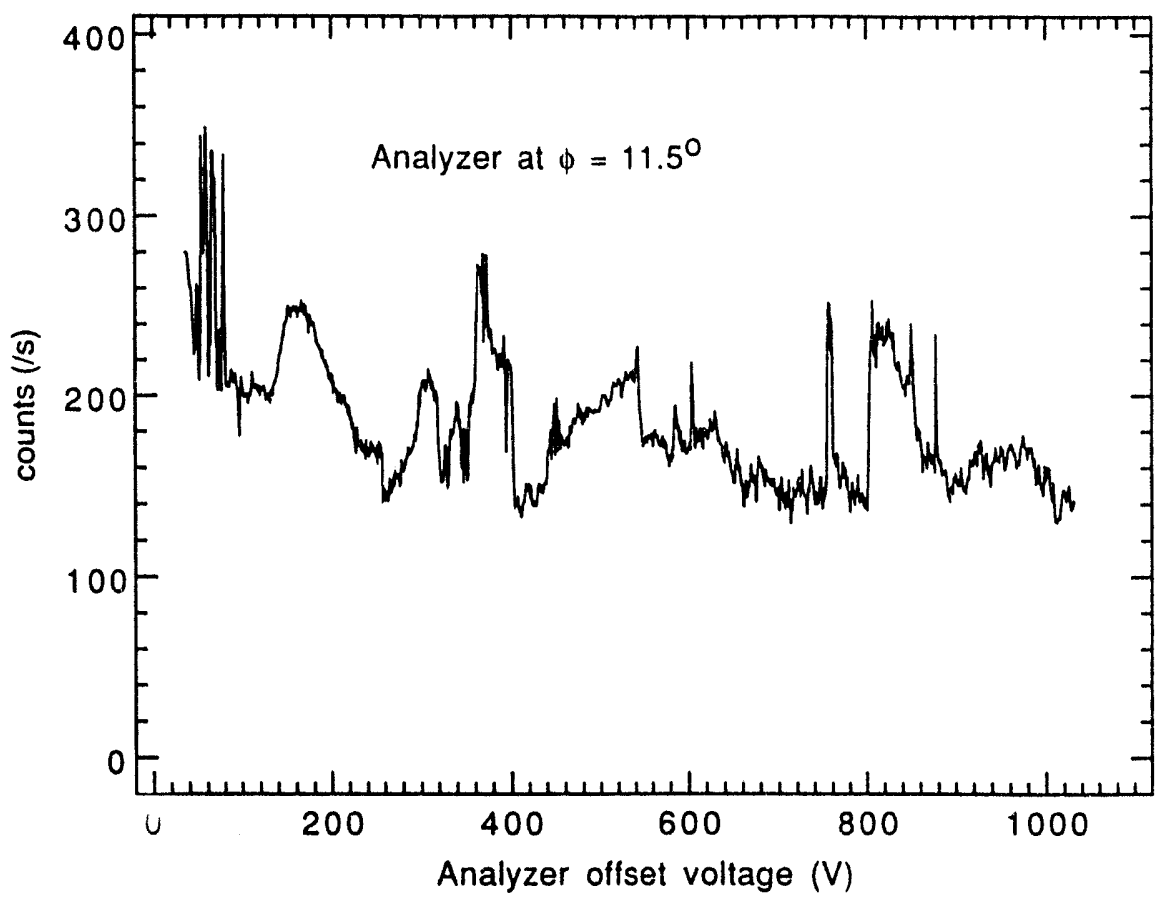

Figure 6. Spectrum of charged particles emitted when $2.3 \mathrm{qkeV} \mathrm{Ar}^{\gamma+}$ ions impinge on a Si $<111>$ crystal. The analyzer channeltron signal is plotted as a function of analyzer offset voltage.

\section{Personnel associated with the project}

The work described in this progress report was carried out mostly by Martin Sadilek, (postdoctoral associate) and myself, Jaroslav Flidr, (graduate research assistant) Jonathan Levine, (undergraduate research assistant) and James Perotti, (research technician).

\section{Publications}

There were no publications during this four and a half month period.

\section{References}

1. E.D. Donets, Sov. J. Part. Nucl. 13, 387 (1982).

2. J. Vancura, J.J. Perotti, J. Flidr, and V.O. Kostroun, Rev. Sci. Instrum. 64, 3139 (1993).

3. "Angular and energy distribution of $\operatorname{Ar}(q-1)+$ in $A r q+(8 \leq q \leq 10)$ on $\operatorname{Ar}$ at laboratory collision energies below 100 qeV" by J. Vancura, M. Sadilek, and V.O. Kostroun, submitted to Phys. Rev. A.

4. V.O. Kostroun, Progress Report DOE/ER/13519-8, April 27, 1994.

5. "Even-odd projectile charge-capture state variation in low energy $\mathrm{Ar} q+(8 \leq q \leq 14) \mathrm{Ar}$ collisions" by J. Vancura and V.O. Kostroun, submitted to Phys. Rev. A 
6. F.C. Fehsenfeld, K.M. Evenson, and H.P. Broida, Rev. Sci. Instum. 36, 284 (1965).

7. E.J. Murphy and J.H. Brophy, Rev. Sci. Instrum. 50, 645 (1979). 

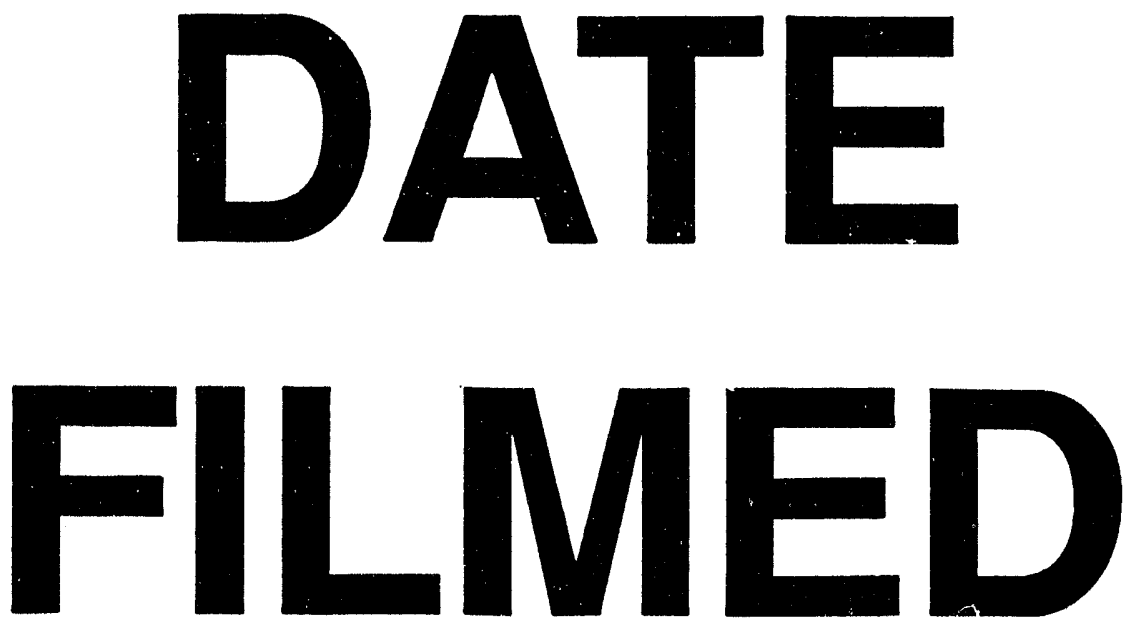

$10 / 17 / 94$
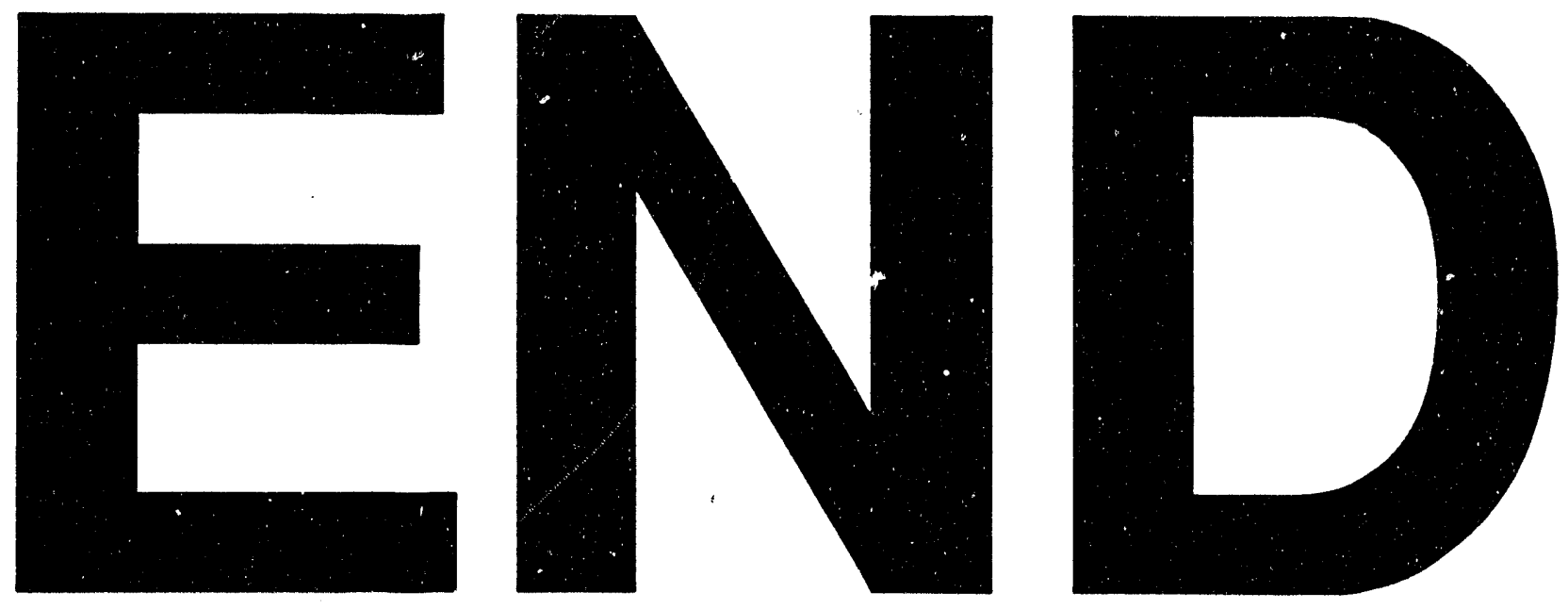
